Rev. Elev. Méd, vét. Pays trop., 1973, 26 (2) : 221-24

\title{
Ostertagia thalae n. sp., parasite d'antilopes d'Afrique Centrale
}

\author{
par P. M. TRONCY $\left({ }^{*}\right)$ et M. GRABER $\left({ }^{* *}\right)$
}

\begin{abstract}
RESUME
Les auteurs décrivent un nouveau Trichostrongylinae qui se caractérise par ses dimensions et, chez le mâle, par ses spicules, le cône génitaI, la côte et le lobe dorsal, et par la membrane bursale accessoire.
\end{abstract}

\section{MATERIEL D'ETUDE}

Au cours de l'étude des Helminthes récoltés chez les animaux de la faune cynégétique de République Centrafricaine (Projet P.N.U.D., C.A.F. 13), un Trichostrongle nouveau a été découvert chez le Bubale et l'Hippotrague. Ses principaux caractères le placent dans le genre Ostertagia, genre dont les subdivisions sont évoquées dans la discussion qui suit la description ci-dessous.

Hôtes: 2 Alcelaphus buselaphus (Pallas, 1766) (Bubale); 1 Hippotragus equinus (Desmarest, 1804) (Hippotrague).

Origine: République Centrafricaine (Coordonnées I.B.A.H. : $218 \mathrm{D} \mathrm{a} ; 218 \mathrm{D} \mathrm{d} ; 246$ B d) $\left({ }^{* * *}\right)$.

\section{Localisation: Abomasum.}

Matériel: Environ 100 vers mâles et femelles chez $A$. buselaphus; 1 mâle et 3 temelles chez $H$. equinus. Holotype et allotype proviennent de $A$. buselaphus.

(*) I.E.M.V.T., Laboratoire de Farcha, B.P. $\pi^{*} 433$, Fort-Lamy, Tchad.

(**) Laboratoire de Parasitologie, Ecole Nationale Vétérinaire, 2, quai Chauveau, 69337 Lyon, Cedex 1, France.

(***) Coordonnées de l'International Bureau of Animal Health (I.B.A.H.), exprimées par des carrés dont les bases sont désignées par la latitude et la longitude.

\section{DESCRIPTION}

Fins Nématodes. Mâles de $7 \mathrm{~mm}$ à $9 \mathrm{~mm}$ de longueur; femelles de 9 à $12 \mathrm{~mm}$. Largeur moyenne dans les deux sexes : $150 \mu$. La cuticule présente des stries longitudinales en relief; elles débutent à $100 \mu$ de l'apex environ, et se terminent au niveau de l'anus ou un peu au-delà chez la femelle; elles sont au nombre de 30 à 50: ce nombre varie tout au long du stroma (fig. 1-I et 1-L). L'extrémité apicale comporte une petite vésicule céphalique de $10-12 \mu$ de hauteur; la cavité buccale est rudimentaire; le plateau céphalique porte 2 amphides et 4 papilles, et son diamètre est d'environ $25 \mu$ (fig. 1-C). La cuticule est striée transversalement sur 20 à $30 \quad \mu$ au-delà de la vésicule céphalique (fig. 1-B). Cette striation peut être discernée parfois entre les lignes cuticulaires longitudinales. L'esophage est formé de 2 parties, musculaire et glandulaire. La partie glandulaire débute au niveau des deirides (fig. 1-A) ou au-delà (à 50-75 $\mu$ environ). Les deirides sont larges et effilées en pointe. Le pore excréteur est au même niveau que les deirides; l'anneau nerveux est un peu en-deçà. Chez la femelle, les 2 utérus sont divergents, les ovéjecteurs sont de dimension variable et la vulve est une ouverture transversale (fig. 1-E et 1-H). Dans un cas, cet organe présentait une ornementation cuticulaire (fig. 1-K). La queue est courte, en forme de massue. L'extrême pointe 


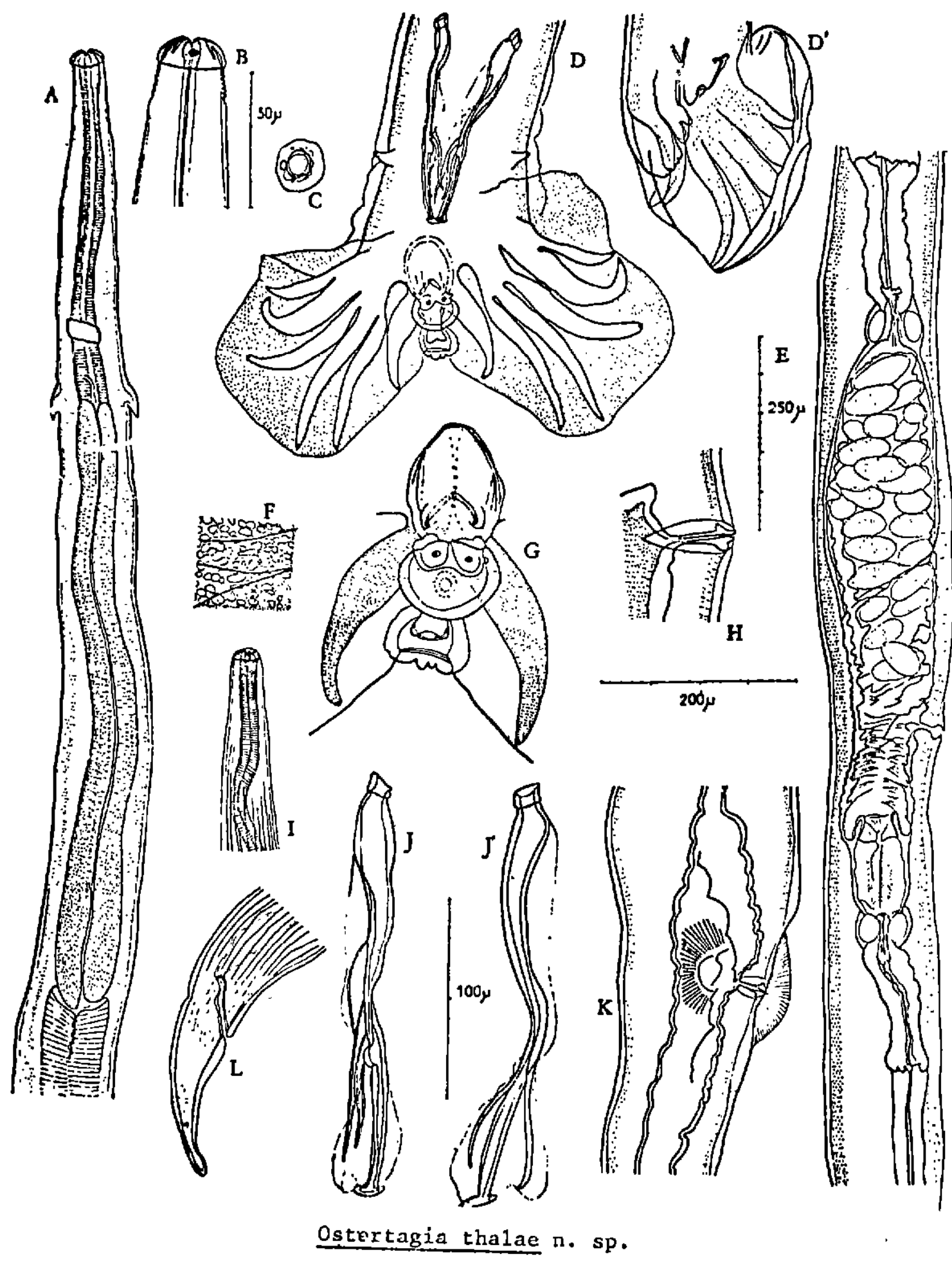

A : Femelle, extrémité antérieure (échelle $200 \mu$ ).

B : Extrémité céphalique (échelle $50 \mu$ ).

C : Vue apicale (échelle $100 \mu$ ).

D-D ${ }^{\prime}$ : Bourse caudale du mâle (échelle $200 \mu$ ).

E : Appareil génital femelle (échelle $250 \mu$ ).

F : Ornementation de la bourse caudale

(échelle $100 \mu$ ).

$\mathrm{G} \quad$ : Cône génital, membrane accessoire et région dorsale de la bourse caudale (échelle $100 \mu$ ). : Région vulvaire de la femelle (échelle $100 \mu$ ). I : Extrémité antérieure: origine des stries longitudinales (échelle $200 \mu$ ).

$\mathrm{J}_{-} \mathrm{J}^{\prime}$ : Spicules (échelle $100 \mu$ ).

K : Dessin montrant l'ornementation de la région vulvaire dune femelle unique (échelle $200 \mu$ ).

L : Femelle, extrémité postérieure (échelle $200 \mu$ ). 
est striée transversalement (fig. 1-L). Chez le mâle, la bourse caudale est précédée de 2 papilles prébursales; sa structure est représentée sur la figure 1-D. Elle est ornementée comme l'indique la figure 1-F. La côte dorsale est courte et ses branches terminales sont réduites à 2 moignons portant chacun une papille (fig. 1-G). Le lobe dorsal est plus ventral que les bords des lobes latéraux et ceux-ci, en se chevauchant, forment une frange de sorte que la côte dorsale semble incluse dans un cône cuticulaire. La membrane bursale accessoire comprend une partie supérieure avec 2 papilles, et une partie inférieure discoïde. Ces structures sont fragiles et difficiles à préciser avec certitude, car certaines parties s'arrachent lorsqu'on ouvre la bourse caudale (fig. 1-G).

Les spicules sont droits de profil, et en $S$ vus de face. Leur extrémité présente trois pointes; la principale se termine par un épaississement en forme de pied; les 2 secondaires, pointues, divergentes, naissent dans le tiers postérieur de l'organe. Pas de gubernaculum, ni de télamon (fig. 1-D, 1-J et 1-J'). Le cône génital, en vue ventrale, est ellipsoïde (fig. 1-G), et présente 2 formations papillaires.

Mâle holotype. - Longueur totale $8,56 \mathrm{~mm}$. Largeur maximale $160 \mu$. Longueur de l'œsophage $960 \mu$, dont portion musculaire $370 \mu$. Largeur maximale de l'œsophage $70 \mu$. Positions par rapport à l'apex de l'anneau nerveux : $270 \mu$, du pore excréteur : $315 \mu$, des deirides : $330 \mu$. Longueur des spicules $200 \mu$. Longueur de la côte dorsale $45 \mu$. Longueur du cône génital $60 \mu$. Longueur de la membrane bursale accessoire: $40 \mu$; largeur maximale de cette membrane : $50 \mu$.

Femelle allotype. - Longueur totale $10,8 \mathrm{~mm}$. Largeur maximale $180 \mu$. Longueur de l'œsophage $910 \mu$, dont portion musculaire $375 \mu$. Largeur maximale de l'œsophage $60 \mu$. Positions par rapport à l'apex de l'anneau nerveux : $245 \mu$; du pore excréteur : $280 \mu$; des deirides : $300 \mu$. La vulve est à $1,8 \mathrm{~mm}$ de l'extrémité caudale. Les deux ovéjecteurs sont constitués chacun par un vestibule, un sphincter et une trompe. Les vestibules ont des longueurs différentes: vestibule antérieur $310 \mu$, vestibule postérieur $250 \mu$ (probablement dû à la quantité d'œufs contenus dans chacun d'eux). Les sphincters mesurent tous les deux $40 \mu$ de long et les trompes $160 \mu$. Eufs intra-utérins : longueur moyenne $70 \mu$, largeur moyenne $40 \mu$.

\section{DISCUSSION}

1. Le genre Ostertagia sensu stricto se caractérise par l'absence de capsule buccale, la didelphic, la pointe caudale simple chez la femelle, la côte dorsale plus ou moins atrophiée chez le mâle.

Par rapport aux autres Trichostrongylinés ayant ces mêmes caractères, il se distingue par la prohéminence des deirides, une striation longitudinale et une faible striation transversale, et, chez le mâle, des spicules courts, divisés, la présence d'un gubernaculum, l'absence de télamon, un cône génital complexe et enfin, la présence d'une membrane bursale accessoire. De nombreux genres très voisins ont été décrits, dont les critères principaux sont des variations sur ces 4 organes: Gubernaculum, télamon, cône génital, membrane bursale accessoire.

Il en a résulté des classifications compliquées, telle celle de SKRJABIN et collab. (1954) (7), qui multiplie les genres sans clarifier la taxonomie. C'est pourquoi nous préférons considérer toutes les unités du «groupe » Ostertagia comme appartenant au genre Ostertagia; tout au plus pensons-nous qu'il $\mathrm{y}$ a avantage à conserver, au moins provisoirement, les divisions actuelles au niveau des sous-genres.

2. Notre matériel se caractérise par les spicules égaux à 3 branches, l'absence du gubernaculum, la présence d'une membrane bursale accessoire et d'un cône génital complexe, et par la côte dorsale qui, sans être atrophiée, a son extrémité distale presque indivise, avec 2 branches réduites à des moignons.

3. Les Ostertagia connus, parasites de ruminants sauvages d'Afrique, sont rares (le genre est aussi pratiquement inconnu chez les ruminants domestiques d'Afrique Centrale: cf. GRABER, 1969) (1). Les sous-genres Spiculopteragia Orloff, 1933, Skrjabinagia (Kassimov, 1942) et Marshallagia Orloff, 1933, ainsi que quelques autres ont une membrane bursale accessoire, et pas de gubernaculum; mais ils ont aussi une côte dorsale, bien dichotomisée à son extrémité distale, qui les différentie tous de nos spécimens.

4. Les espèces africaines voisines appartiennent aux genres Longistrongylus Leroux, 1931, 
Kobusinema Ortlepp, 1963 et Bigalkenema Ortlepp $1963\left(^{*}\right)$. Elles sont dépourvues de membrane bursale accessoire. (Le genre Longistrongylus a été rencontré au Tchad par GRABER (1964) (2) chez Hippotragus equinus, Alcelaphus lelwel et chez Kobus defassa.; le genre Kobusinema chez $H$. equinus.)

Notre matériel est donc différent des espèces

(*) A noter que Bigalkenema namaquensis Ortlepp, 1963 , montre un œsophage en deux parties. Ce caractère se retrouve chez $O$. (Spiculoptesagia) trmitatis (Cameron, 1935) Travassos, 1937. et même des sous-genres connus chez Ostertagia, et constitue une espèce nouvelle. Pour ne pas alourdir inutilement la nomenclature déjà compliquée du genre, nous ne pensons pas toutefois utile de créer un sous-genre nouveau pour le classer. C'est pourquoi nous proposons simplement de le désigner: Ostertagia thalae n. sp., en hommage au Docteur Jean THAL, qui l'a récolté.

Les spécimens types sont conservés au $\mathrm{Mu}-$ séum national d'Histoire naturelle sous les $\mathrm{n}^{\text {is }} 43 \mathrm{MA}, 44 \mathrm{MA}, 45 \mathrm{MA}$ et $46 \mathrm{MA}$ - Bocal N 304.

\section{SUMMARY}

\section{Ostertagia thalae n. sp., a parasite of Central African antelopes}

The authors describe a new Trichostrongylinae characterised by its dimensions, and, in the male, by the spicules, genital cone, dorsal rib and dorsal lobe, and by the accessory bursal lobe.

\section{RESUMEN}

\section{Ostertagia thalae n. sp., parásito de antilopes de Africa Central}

Los autores describen un nuevo Trichostrongylinae cuyas caracteristicas son sus dimensiones y. en el macho, sus espículas, el cono genital, la costilla y el lóbulo dorsal, y la membrana bursal accesoria.

\section{BIBLIOGRAPHIE}

1. GRABER (M.). Helminthes parasites de certains animaux domestiques et sauvages du Tchad. Bull. epizoot. Dis. Afr., 1969, 17 (4) : 403-428.

2. GRABER (M.), DOUTRE (M.), FINELLE (P.), KERAVEC (J.), DUCROZ (G.) et MOKOTAINGAR ( $\left.P_{1}\right)$. Les helminthes de quelques artiodactyles sauvages appartenant aux familles des bovidés et des suidés. Ces mammifères, en République du Tchad et en R.C.A., sont-ils des réservoirs de parasites pour les animaux domestiques vivant à leur contact? Rev. Elev Méd.vét. Pays trop., 1964, 17 (3) : $377-420$.

3. LE ROUX (P. L.). On Longistrongylus meyeri gen. et sp. nov., a trichostrongyle parasitizing the Red Hartebeest, Bubalis caama. J. Helminth. 1931, 9 (3) : 141-146.

4. ORLOFF (I. V.). Sur la reconstruction de la systé- matique du genre Ostertagia Ransom, 1907 - Ann. Parasit. hum. comp., 1933, 11 (2) : 96-114.

5. ORTLEPP (R. J.). Bigalkenema namaquensis gen. et sp. nov., a trichostrongylid worm from sheep. Onderstepoort J. vet. Res., 1963, 30 (1) : 119-123.

6. ROUND (M. C.). Check list of the helminth parasites of African Mammals. St Albans, Commonwealth Bureau of Helminthology, 1968, Technical communication $\mathrm{n}^{\circ} 38,252 \mathrm{p}$.

7. SKRJABIN (K. I.), SHIKHOBALOVA (N. P.) et SCHULTS (R.S.). Essential of nematodology. III. Trichostrongylids of animals and man. Acad. Sci. U.R.S.S. Moscou, 1954. (Traduction anglaise publiée par «Israël Program for scientific Translations $», 1960,704$ p.)

8. TRAVASSOS (L. P.). Revisão da familia Trichostrongylidae, Leiper, 1912. Monogr. Inst. Oswaldo Cruz. 1937, no $^{\circ} 1,512$ p.). 